\section{Paracentesis for successful treatment of tension pneumoperitoneum related to endoscopic sub- mucosal dissection}

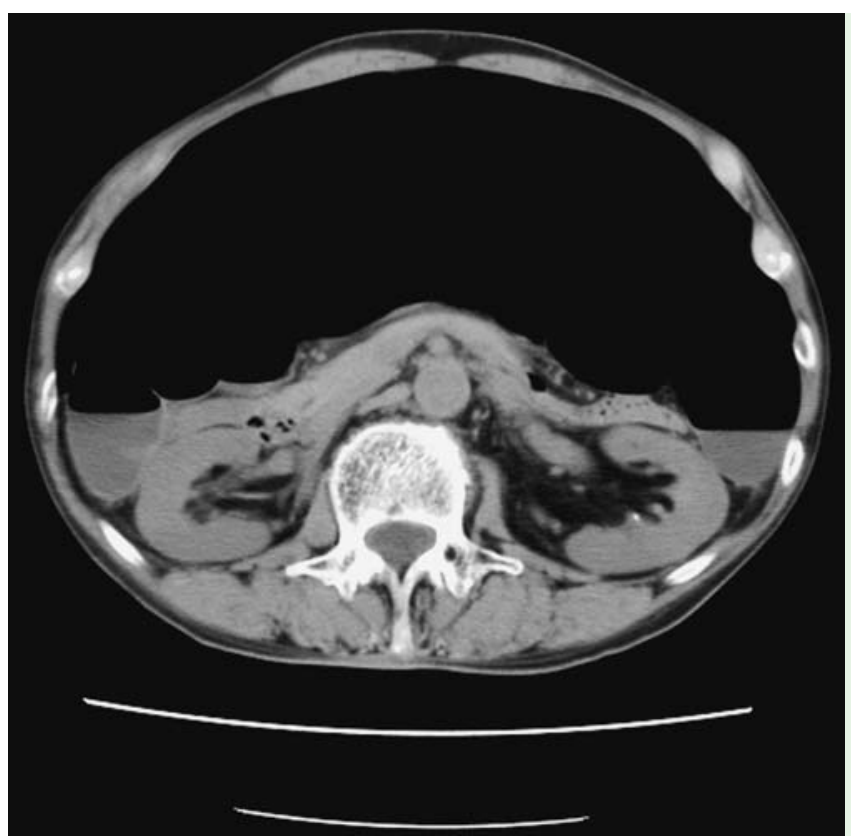

Fig. 1 Abdominal computed tomography showed a large pneumoperitoneum causing collapse of the inferior vena cava.

A 78-year-old man underwent total colonoscopy because of positive fecal occult blood test. Two lateral spreading tumors (LST), approximately $35 \mathrm{~mm}$ and $40 \mathrm{~mm}$ in size, were detected in the cecum and transverse colon, respectively. Both lesions were endoscopically diagnosed as adenoma or focal cancer in adenoma, and therefore considered good candidates for endoscopic resection.

Initially, endoscopic submucosal dissection (ESD) was attempted for en bloc resection of the LST located in the cecum. During the procedure, although no definite perforation was recognized, the patient became aware of marked abdominal distension, and then developed hypotension, his blood pressure decreasing to $80 \mathrm{~mm} \mathrm{Hg}$. The procedure was immediately discontinued, and emergency abdominal computed tomography (CT) demonstrated a large pneumoperitoneum with collapse of the inferior vena cava ( Fig. 1). Paracentesis was performed for decompression with a 20 gauge puncture needle. The patient's symptoms were relieved, and the blood pressure and diameter of the inferior vena cava returned to normal immediately after decompression. The patient recovered uneventfully after conservative treatment, including withholding oral intake, intravenous administration of antibiotics, and hyperalimentation.

In order to completely remove large colorectal tumors endoscopically en bloc, a novel technique of ESD instead of conventional endoscopic mucosal resection (EMR) has been developed [1]. This technique is reportedly associated with a significant incidence of complications, such as perforation and bleeding, in comparison with conventional EMR [1,2]. However, most perforations that occur during ESD are small and can be managed conservatively with endoclip placement if recognized immediately. In the present case, the exact site of perforation, which may have been a mini-perforation, could not be detected endoscopically, and thus pressurized air resulted in increased intra-abdominal pressure. In general, increased intra-abdominal pressure can have numerous adverse physiologic effects, which may include decreased venous return, decreased cardiac output, and altered ventilation-perfusion relationships $[3,4]$. Tension pneumoperitoneum should be suspected in all patients who develop circulatory and/or respiratory collapse with acute abdominal distension during or after endoscopy, espe- cially for treatments that carry a high risk of perforation [3]. Early identification and prompt treatment with needle decompression are important in order to avoid serious sequelae such as abdominal compartment syndrome leading to multiple organ failure [4].

\section{Acknowledgment \\ $\nabla$}

This work was supported in part by the Grant-in-Aid for Cancer Research (18S-2, H18-005) from the Ministry of Labour and Welfare.

Endoscopy_UCTN_Code_CPL_1AJ_2AC Endoscopy_UCTN_Code_TTT_1AO_2AG

\section{K. Fu ${ }^{1,2}$, T. Ishikawa2, T. Yamamoto², Y. Kaji ${ }^{1}$ \\ Department of Radiology, Dokkyo Medical University, Tochigi, Japan \\ 2 Department of Diagnostic Imaging, Division of Endoscopy, Tochigi Cancer Center Hospital, Tochigi, Japan}

\section{References}

1 Sano Y, Machida H, Fu KI et al. Endoscopic mucosal resection and submucosal dissection method for large colorectal tumors. Dig Endosc 2004; 16: S88 - S91

2 Fujishiro M, Yahagi N, Nakamura M et al. Endoscopic submucosal dissection for rectal epithelial neoplasia. Endoscopy 2006; 38: 493-497

3 Yakobi-Shvili R, Cheng D. Tension pneumoperitoneum - a complication of colonoscopy: recognition and treatment in the emergency department. J Emerg Med 2002; 22: 419-420

4 Peppriell JE, Bacon DR. Acute abdominal compartment syndrome with pulseless electrical activity during colonoscopy with conscious sedation. J Clin Anesth 2000; 12: 216-219

Bibliography

DOI 10.1055/s-2007-966489

Endoscopy 2009; 41: E245

(c) Georg Thieme Verlag KG Stuttgart · New York . ISSN 0013-726X

\section{Corresponding author}

\section{K. Fu, MD}

Department of Radiology

Dokkyo Medical University

880 Kitakobaysashi

Mibu

Shimotuga

Tochigi 321-0293

Japan

Fax: +81-282-865678

fukuangi@hotmail.com 